\title{
Revisión de la Literatura: Déficit de Procesamiento Sensorial en el Espectro del Autismo
}

\section{Literature Review: Sensory Processing Deficits in Autism Spectrum Disorder}

\author{
Erna Imperatore Blanche, PhD, OTR/L, FAOTA ${ }^{1}$ \\ Gustavo Reinoso, $\mathrm{PhD}, \mathrm{OTR} / \mathrm{L}^{2}$
}

\begin{abstract}
Resumen (español)
En muchas oportunidades los problemas sensoriales presentados por niños con autismo son confundidos con conductas aprendidas sin base biologica. Este articulo es una revisión de la literatura que trata con el tema de deficit sensoriales en inidivuos con autismo. La literatura que describe estos deficits sensoriales se puede dividir en tres tipos de estudios: colección de conductas a traves de cuestionarios, estudios neuro-fisiologicos y autobiografias. El deficit sensorial en individuos con autismo esta presente en la mayoría de los casos e incluye multiple sistemas sensoriales. Este deficit debe ser identificado y tratadosen el proceso de intervención.
\end{abstract}

\footnotetext{
${ }^{1}$ Profesor Asociado de Práctica Clínica División de Ciencia de la Ocupación y Terapia Ocupacional, Universidad Southern California. USA. Profesor Asociado de la Escuela de Terapia Ocupacional, Facultad de Medicina. Universidad de Chile. Co-Directora de Therapy West, Los Angeles California, USA. Impe@USC.edu

2 Profesor Asociado de Práctica Clínica División de Ciencia de la Ocupación y Terapia Ocupacional, Universidad Southern California. USA.gustyot@yahoo.com
} 
Palabras claves: Trastorno del espectro autista, Integración sensorial, Desorden del procesamiento sensorial

\begin{abstract}
English)
In many opportunities the sensory processing deficits presented by children with autism are misinterpreted as behavioral issues with no biological base. This article is a review of the literature describing sensory processing deficits in children with autism. The literature reviewed can be divided into three types of studies: data collection through questionnaires, studies focusing on the neurofisiological aspects of sensory processing, and autobiographical accounts. The majority of individuals diagnosed with autism present sensory processing deficits that cut accross many sensory systems. These deficits need to be identified and addressed in the intervention process.

Palabras claves: Espectro del autismo, integracion sensorial, deficits de proceso sensorial Keywords: Autism Specturm Disoder, sensory integration, sensory processing disorders
\end{abstract}

\title{
Introducción:
}

Autismo es un patrón de retraso del desarrollo de aspecto biológico y etiológico diverso (Herbert, 2005). El trastorno generalmente esta caracterizado por un comienzo temprano de déficit cualitativos en la interacción social y conductas estereotipadas que también pueden manifestarse en el sistema inmune, gastrointestinal, y de las funciones sensoriales (Greenspan, 2006; Herbert, 2005). Autismo, Asperger (AS) y Trastornos Profundos del Desarrollo (PDD) constituyen el Espectro del Trastorno del Autismo (ASD) (American Psychiatric Association, 2000). El diagnostico del espectro esta basado primordialmente 
en observaciones de la conducta, chequeos o entrevistas realizados por profesiones con experiencia (Volkmar et al, 2005). En el presente ASD es uno de los trastornos del desarrollo que están creciendo mas rápidamente en USA y otras partes del mundo. La asociación de autismo de USA (The Autism Society of America, 2006) reporta que en el ano 2006 existían 1.5 millones de individuos diagnosticados con autismo en USA, un aumento del $170 \%$ en la prevalencia de este diagnostico desde 1990 . Las estadísticas indican que 1 en 166 (150) de los nacimientos vivos son niños diagnosticados con autismo. Se estima que el costo anual de intervención con estos niños en USA es de 90 billones de dolares de los cuales el $90 \%$ se gasta en el cuidado del adulto (Autism Society of America, 2006). Dos tercios de esta cantidad podría ser reducida con diagnostico e intervención temprana (Autism Society of America, 2006)

El proceso de diagnostico del ASD durante los primeros anos de edad sigue siendo un desafió para los padres y los profesionales. La literatura es rica en estudios que describen los primeros signos del autismo, pero estos estudios no son usados en la practica clínica y las familias viven con la intercidumbre en vez de la confirmación que las conductas que exhiben los niños son signos de un trastorno severo en el desarrollo (Schieve, Blumberg, Rice, Visser \& Boyle, 2007).

Central en el diagnostico temprano del autismo son los signos de disfunción sensorial presentados por estos niños, los cuales muchas veces llevan a los padres a la búsqueda de consulta profesional. Signos de disfunción sensorial en niños con diagnostico de autismo han sido descriptos tempranamente en la literatura. Kanner (1943) describió no solo la fascinación y el placer que los niños con diagnostico de autismo experimentan en relación a ciertos estímulos sensoriales como el reflejo de la luz en los espejos sino 
también signos asociados con estrés como por ejemplo el cubrirse los oídos en presencia de ciertos sonidos. La descripción de signos de déficits en el procesamiento sensorial en el niño con diagnostico de autismo abunda en la literatura e incluyen no solo el resultado de estudios de investigación y reportes clínicos sino también numerosas descripciones cualitativas de carácter biográfico (Bemporad, 1979; Cesaroni and Garber, 1991; Grandin and Scariano, 1986; Gerland, 2003; Jones, Quigney, \& Huws, 2003; Williams, 1999) Terapistas ocupacionales y físicos muchas veces reciben a estos niños en su practica clínica y se encuentran en la situación de escoger la intervención ideal para tratar estos problemas. Este articulo busca elucidar la evidencia en la literatura de investigación que describe los problemas sensoriales presentes en el diagnostico de autismo para que de esta manera el profesional tenga claro que estos déficit impactan el funcionamiento ocupacional de estos niños y de la familia.

La revisión de la literatura se condujo del siguiente modo. Bases de datos comúnmente utilizadas por la comunidad científica fueron revisadas sistemáticamente en busca de publicaciones cuyo énfasis principal fuera la descripción de los problemas sensoriales en los individuos con diagnostico de autismo. La búsqueda incluyo artículos cuyo titulo o resumen incluyera los descriptores autismo y sus sub-descriptores, sensorial y subdescriptores y que fueran publicados en el periodo 1997-2007 en el idioma ingles. Técnicas de reducción en el volumen de la literatura se emplearon considerando disponibilidad, relevancia, y validez. Las listas de referencias enumeradas en los artículos revisados se consideraron en relación a su aporte al tema de revisión. Bases de datos utilizadas incluyeron Ovid databases (United States National Library of Medicine, 2007), Health and Psychosocial Instruments (Behavioral Measurement Database Services, 2007) 
Medline (United States National Library of Medicine, 2007), Psychinfo (Cambridge Scientific Abstracts, 2007), Eric (Eric, 2007), Mantis (The Action Potential Corporation, 2007), First Search (United States National Library of Medicine, 2007), OT Search (OT Search, 2007), OT Seeker (OT Seeker, 2007). Esta revision esta basada en articulos que pueden proporcionar mas evidencia al trabajo de terapeutas ocupacionales.

\section{Resultados de la revisión}

Los resultados de la revision apoyaron la presencia de disfunciones sensoriales en niños con autismo. La mayoria de los estudios se centran en los sistemas auditivos y táctiles y mas que nada en disfunciones de hyper o hypo respuesta mas que en disfunciones de discriminación.

Es relevante establecer que los síntomas sensoriales presentes en varios de los sistemas descriptos en la literatura en el individuo con autismo no son atribuidos a déficit en la agudeza (por ejemplo visual o auditiva) del mismo individuo (Rosenhall, Nordin, Sandstroem, Ahlsen, \& Gillberg, 1999; Scharre \& Creedon, 1992). Actualmente deficits sensoriales no son necesarios para el diagnostico de autismo y generalmente son explicados bajo el rubro de características asociadas (Happe, 1995)

Dentro de los signos mas comúnmente reportados en la literatura se encuentran patrones de hyporrespuesta e hyperrespuesta. La coexistencia de ambos patrones (hypo e hyperrespuesta) tambien ha sido descripta en individuos con diagnostico de autismo (Greenspan \& Wieder, 1997; Hirstein et al., 2001). Además, basado en evidencia del Perfil Sensorial (Dunn, 1999) se describe a niños que son buscadores de sensaciones (Liss, et al. 2006). Esta ultima categorización esta menos clara y podría se parte de las categorizaciones anteriores. La tabla 1 describe las características esenciales de los 
patrones de déficit relacionados al procesamiento sensorial en el niño con diagnostico de autismo.

Tabla 1. Patrones de disfunción, sus características y ejemplos de evidencia en el niño con diagnostico de autismo.

\begin{tabular}{|c|c|c|}
\hline Patrón & Características & Ejemplos de evidencia \\
\hline Hypo respuesta & $\begin{array}{l}\text { Se refiere a la falta de respuesta o a una } \\
\text { respuesta de insuficiente intensidad al los } \\
\text { estímulos sensoriales. }\end{array}$ & $\begin{array}{l}\text {-Disminución de la respuesta } \\
\text { al dolor (Baranek et al., 2006) } \\
\text {-Falta de orientación a sonidos } \\
\text { novedosos (Baranek et al. } \\
\text { 2006) }\end{array}$ \\
\hline Hyper respuesta & $\begin{array}{l}\text { Se refiere a una respuesta } \\
\text { comportamental exagerada a los } \\
\text { estímulos sensoriales } \\
\text { Arousal alto y habitación baja }\end{array}$ & $\begin{array}{l}\text {-Reacción de aversión a la luz } \\
\text { (Baranek, 2006) } \\
\text {-Evitación de las experiencias } \\
\text { táctiles (Miyasaki, 2007; } \\
\text { Miller et al., 2001; Baranek et } \\
\text { al. 2006;) } \\
\text {-Molestias en presencia de } \\
\text { sonidos } \\
\text { Chawarska, \& Klin, 2005; } \\
\text { Baranek, Foster \& Berkson, } \\
\text { 1997) }\end{array}$ \\
\hline $\begin{array}{lr}\text { Patron } & \text { mixto } \\
\text { (hypo e } & \text { hyper } \\
\text { respuesta) } & \end{array}$ & $\begin{array}{l}\text { Combinación en las respuestas } \\
\text { comportamentales de hypo e hyper } \\
\text { respuesta }\end{array}$ & $\begin{array}{l}\text {-Variabilidad exagerada en el } \\
\text { patrón de respuesta a la } \\
\text { información sensorial } \\
\text { (Greenspan and Wieder, 1997; } \\
\text { Hirstein et a., 2001) }\end{array}$ \\
\hline Praxis deficits & $\begin{array}{lrrr}\text { Se refieren a dificultades en } & \text { la } \\
\text { conceptualizacion, } & \text { organización } & \text { y }\end{array}$ & $\begin{array}{llr}\text {-Imitación } & \text { de } & \text { gestos, } \\
\text { imitacion de gestos al }\end{array}$ \\
\hline
\end{tabular}




\begin{tabular}{|l|l|l|}
\hline & $\begin{array}{l}\text { ejecucion de una secuencia de acciones } \\
\text { no habituales }\end{array}$ & $\begin{array}{l}\text { comando verbal, utilizacion de } \\
\text { herramientas (Mostofsky et } \\
\text { al., 2006) }\end{array}$ \\
\hline
\end{tabular}

Consideraciones Etiológicas relacionadas a la disfunción sensorial en individuos con autismo

Numerosas especulaciones existen en relación al origen de los déficit en el procesamiento sensorial que experimentan los individuos con diagnostico de autismo y trastornos relacionados. Estas especulaciones están basadas en diferentes hipótesis de disfunción como factor causal e incluyen problemas de modulación de los estímulos sensoriales (Ornitz, 1989); déficit en los procesos de discriminación sensorial (Tecchio et al., 2003); elevados niveles de alerta (Hirstein et al., 2001); atención selectiva (Kinsbourne, 1987); déficit en los procesos de entrada (gating) sensorio motriz (McAlonan et al., 2002); desequilibrio en los procesos de excitación/inhibición en sistemas neurales claves (Rubenstein \& Merzenich, 2003); y canalesthesia (Waterhouse, Fein, \& Modahl, 1996).

\section{Tipos de estudios}

Los estudios de déficit sensoriales en niños con autismo se han basado en tres métodos: cuestionarios, estudios neurofisiológicos y reportes biográficos proporcionados por personas con diagnostico de autismo. 


\section{Cuestionarios}

Los cuestionarios incluyen la Entrevista Diagnostica para los Trastornos Sociales y de la Comunicación (Leekam et al., 2006); el Cuestionario de Sensitividad Sensorial (TalayOngan \& Wood, 2000); la Agenda de Comportamientos Sensoriales (Harrison \& Hare, 2004); la Evaluación del Procesamiento Sensorial, traducido al español pero no publicado en español (Parham \& Johnson-Ecker, 2000), el Perfil Sensorial publicado en español (Dunn, 1994), la versión breve del Perfil Sensorial (Dunn, 1999), y el Cuestionario de la Experiencia Sensorial sin traducción conocida al español (Baranek et al., 2006). Estos instrumentos trazan un perfil de las reacciones del individuo con diagnostico de autismo en diferentes situaciones $\mathrm{y}$ en respuesta a estímulos sensoriales ambientales. Es interesante destacar que estos instrumentos han sido sometidos a gran escrutinio científico en relación a sus propiedades psicometrías. La investigación por medio de estos cuestionarios ha demostrado la naturaleza de los patrones sensoriales de hipo e hyper respuesta (Kern et al., 2006; Leekam et al., 2006; O’Riordan \& Passetti 2006; Rogers, and Ozonoff, 2005), una prevalencia de disfunción sensorial en 90\% de los individuos con autismo (Leekam et al., 2006), correlación con diferentes niveles del desarrollo siendo en muchos casos uno de los síntomas del autismo que se detectan mas temprano (Baranek et al., 2006); y su habilidad para diferenciar entre poblaciones con autismo y otros diagnósticos (Baranek et al., 2006; Dunn 1999; Leekam et al., 2006; O’Riordan and Passetti, 2006).

Entre estos estudios Kientz and Dunn (1997) compararon a niños con y sin autismo en las respuestas dadas por los padres al Perfil Sensorial (Dunn, 1999). Los resultados 
demostraron que $85 \%$ de las respuestas dadas en este cuestionario diferenciaban al niño con autismo de niños sin autismo, de esta manera apoyando la hipothesis de que niños con autismo tiene déficits de tipo sensorial. Kern et al. (2006) reportan déficit en los sistemas auditivo, táctil, y procesamiento oral que difieren significativamente en comparación con sujetos de control. Tomchek \& Dunn (2007) estudiaron a 281 niños con autismo y sus controles, niños que se desarrollaban normalmente y encontraron que $92 \%$ de los ítems de la version breve del Perfil Sensorial (Dunn, 1999) diferenciaba entre niños con y sin autismo y las diferencias mas grandes estaban en las secciones de baja respuesta/busca sensaciones, bajo filtro auditivo y sensitividad táctil. Otro estudios reportan déficit sensoriales principalmente en el sistema táctil, olfativo, gestatorio y visual sugiriendo un déficit que incluye muchas modalidades y que esta presente a lo largo del desarrollo (Leekam et al, 2006).

Por otro lado, Barankek et al. (2006) utilizando no solo un cuestionario sensorial pero también análisis retrospectivos de videos del primer ano de edad indica que niños con autismo presentan patrones de hypo e hyerrespuesta sensorial que no solamente estan ligados a interacción social. Hasta el presente no es claro si la edad cronológica esta relacionada al comienzo de las disfunciones sensoriales presentes en el individuo con autismo (Baranek, 1999a; Stone \& Hogan, 1993), o si estas características se incrementan (Talay-Ongan \& Wood, 2000), disminuyen (Baranek et al., 1997) o se modifican (Rogers et al., 2003) como resultado de los procesos de maduración y desarrollo.

En general, estos estudios demuestran que niños con autismo tienen déficit sensorial y que el déficit sensorial puede aparecer en todos los sistemas incluyendo los sistemas vestíbulo/proprioceptivo pues dentro de los ítems de baja respuesta/busca sensaciones en 
los cuestionarios sensoriales existen ítems de tipo vestibular/proprioceptivo. Además, los patrones de respuesta sensorial de niños con autismo distinguen este grupo de otros diagnósticos en que la prevalencia de disfunción sensorial es mas alta en niños con autismo que en otros niños con desordenes o retraso en el desarrollo (Leekam et al, 2006).

\section{Estudios neurofisiológicos}

Aunque las disfunciones de procesamiento sensorial son observables en la clínica y se reportan en cuestionarios, su relación a procesos neurofisiológicos es menos clara. En los últimos años se ha aumentado el interés en el estudio de la relación entre patrones de conducta y mediciones neurofisiológicas. Entre ellas se encuentran los estudios de actividad electrodérmica (Galvanic Skin Response), estudios de saliva, EEG, estudios de potenciales evocados y estudios de imágenes.

Los estudios de actividad electrodérmica miden las respuestas a input sensorial a través de la conducción a nivel de la piel en respuesta a actividad del sistema nervioso simpático. Las mediciones que se pueden hacer a través de este sistema incluyen: respuesta tónica o durante reposo (sin estímulos), respuesta a input sensorial (reactividad al input), y habituación al input. Estas mediciones se llevan a cabo dentro de un ambiente controlado. Los resultados de estudios que usan estas mediciones aun no llegan a un acuerdo. En general se ha demostrado que niños con autismo tienden a una hiper respuesta y pobre habitación a input auditivo (Barry \& James, 1988; James \& Barry, 1984). Mas recientemente Miller y colaboradores desarrollaron el protocolo de desafió sensorial (Sensory Challenge Protocol) para medir respuestas del sistema simpático y 
parasimpático a input sensorial (Miller, et al., 1999; Miller, et al., 2001). Los resultados preliminares muestran déficit sensoriales en niños con autismo

Estudios de cortisol a través de la saliva se han utilizado para medir el stress de niños con autismo al encontrarse con situaciones novedosas. Estos estudios han medido respuestas en el sistema hipotalamico-pituitario-adrenocortical (HPA) y han demostrado estadisticamente niveles mas elevados de cortisol en ninos con autismo en comparacion con controles (Corbet et al., 2006).

Estudios de potenciales evocados y otros procedimientos especificos se han utilizado para medir el proceso de inputs auditivos (Tharpe et al., 2006) y somatosensoriales (Miyasaki et al., 2007). Alguna evidencia de estudios fisiológicos auditivos parecen demostrar que niños con autismo obtienen resultados equivalentes a niños control en audición periférica (Gravel et al., 2006) pero no en mediciones relacionadas a variables comportamentales (Tharpe et al., 2006), material dinámico o tareas auditivas complejas medidos con potenciales relacionados a eventos (Event Related Potentials o ERP) que muestran patrones de activación cerebral inferiores (Samson et al., 2006). Algunos de estos estudios sugieren problemas relacionados a hypersensitividad auditiva, habilidades adecuadas para el procesamiento de entonacion, discriminacion auditiva disminuida y dificultades con orientacion relacionada a sonidos sociales (Lepisto et al., 2005). Resultados similares han sido reportados en adultos con diagnostico de autismo (Kasai et al., 2005). En el sistema somatosensorial Miyasaki y colaboradores estudiaron potenciales evocados somatosensoriales por medio de estimulacion del nervio mediano derecho e izquierdo. Estos autores reportaron que casi la mitad de los ninos con autismo 
estudiados presentaron anormalidades asociadas a hyperactividad hemisferica derecha, en comparacion con la izquierda, especialmente en el area somatosensorial primaria.

Evidencia derivada de estudios que emplean resonancia magnetica funcional (fMRI) y ERP han revelado resultados similares con patrones de activacion reducidos en la region temporo parietal $\mathrm{y}$ frontal conocidas por su rol en cambios atencionales y en la distribucion de los recursos de atencion en relacion a eventos novedosos (Gomot et al., 2006). Similarmente, evidencia relacionada a la percepcion visual parecen apuntar a difficultades en el procesamiento sensorial. Por ejemplo el procesamiento de información relacionada a la cara parece encontrar apoyo en los estudios de neuroimagen señalando aspectos de deficits en el procesamiento visual en niños con autismo, independientemente de la función social (Davis, et al. 2006; Behrmann et al., 2006).

\section{Estudios biográficos}

Entre los estudios biográficos de personas con autismo el mas importante es el de Temple Grandin (Grandin and Scariano, 1986) que describe los problemas sensoriales que sentía desde pequeña. En este recuento, ella describe su necesidad por presión e input vestibular de tal forma que la llevan a construir equipo proveedor de presión que se ocupa en clínicas de terapia ocupacional (Eldenson, Edelson, Kerr, and Grandin, 1999). Otros recuentos biográficos describen experiencias similares en relacion a la dificultad en el procesamiento de información sensorial (Bemporad, 1979; Cesaroni and Garber, 1991; Gerland, 2003; Jones, Quigney, \& Huws, 2003; Williams, 1994, 1999)

\section{Síntesis de los problemas sensoriales en niños con autismo}

Los estudios basados en cuestionarios apoyan la idea que niños con autismo tienen un déficit de procesamiento sensorial que tiende a ser multisensorial y mas bien de 
hyporespuesta. Los resultados de estudios fisiológicos son más variados. La presencia de disfunciones auditivas esta probada por algunos estudios y no por otros, y los estudios de disfunciones táctiles y vestíbulo/proprioceptivos están recién empezando. Los resultados apuntan a una hyper respuesta y un alto nivel de alerta y stress. Esta conclusión no encuentra apoyo en un estudio de la literatura realizado en el ano 2005 en el cual los autores revisaron 48 estudios de laboratorio y concluyen que existe evidencia que apoya la presencia de déficit sensorial en niños con autismo. Estos déficit sensoriales son de tipo de hipo respuesta y que existe poco apoyo para la presencia de falta de habituación y estados de hyper alerta en niños con autismo (Rogers, \& Ozonoff, 2005).

En conclusión, aunque los artículos revisados no concuerden en el tipo de disfunción sensorial presentada por niños con autismo, los estudios concuerdan en la presencia de una disfunción de tipo sensorial. Es mas, muchos de ellos concuerdan en la contribución de disfunciones sensoriales a los problemas posturales y de praxis presentados por los niños con este diagnostico La presencia de esta disfunción sensorial apoya el uso estrategias sensoriales en la intervención.

\section{Aplicación a la Practica Clínica y Conclusión}

La importancia de entender los déficit sensoriales en niños con autismo esta en el efecto que estas disfunciones tienen en su funcionamiento. Los problemas sensoriales presentes en niños con autismo han sido descriptos en relación a su efecto en la atención, la presencia de conductas estereotipadas, la praxis que consiste no solo en pobre imitación sino también en una pobre representación sensorial del moviendo y su secuencia motora 
(Mostofsky et al., 2006) y los déficit posturales cuando el input somatosensorial se encuentra alterado (Minshew, Sung, Jones, \& Furman, 2004).

El estudio sistemático de disfunciones sensoriales en niños con autismo esta comenzando, sus resultados apuntan a déficit que como terapistas ocupacionales debemos identificar especialmente cuando tratamos a niños pequeños pues podría ser uno de los signos del autismo que se identifican mas tempranamente.

Dentro de la literatura existen también estudios que describen los resultados obtenidos cuando se usa input sensorial durante la intervención. Con la excepción del estudio de Ayres y Tickle (1981) estos estudios no identifican la disfunción sensorial inicial del niño. El estudio original de Ayres y Tickle indica que niños que son hyper responsivos responden mejor a tratamiento de IS que niños que no registran o son hipo responsivos a la información sensorial. Hay que tomar estos resultados con precaución ya que el diagnostico de autismo ha cambiado mucho en los últimos 20 anos indicando que este estudio debe repetirse. Otros estudios de casos sugieren que el uso de input vestibular en la sesión aumenta la comunicación a través del lenguaje y contacto visual (Karsteadt, 1983; Linderman \& Steward, 1999; Ray, King, \& Grandin, 1988). Otros estudios que existen en tratamiento muestran como la utilización de experiencias sensoriales adecuadas disminuyen las conductas estereotipadas y aumentan la atención (Zisserman, 1992; Smith, Press, Koenig, \& Kinnealey, 2005). Estudios que también apoyan el uso de estrategias sensoriales para aumentar la atención y la participación son estudios que utilizan chaqueta con pesos (Fertel-Daly, Bedell, Hinojosa, 2001) o presion con la maquina de Grandin (Edelson et al, 1999). 
En conclusión el uso de la investigación de déficits sensoriales en niños con autismo indica que como terapeutas ocupacionales debemos realizar evaluaciones sistemáticas de los déficit sensoriales del niño. Estas evaluaiones deben examinar cada uno de los sistemas sensoriales por separado y en conjunto, además debemos entender la importancia de esos déficit sensoriales en el funcionamiento diario del niño, antes de decidir que experiencias sensoriales utilizar como parte de la intervención. Solo evaluando el déficit sensorial al comienzo de la intervención y midiendo los resultados de nuestro manejo de input sensorial podemos tratar a estos niños en forma eficaz.

\section{Referencias}

- American Psychiatric Association (2000). Diagnostic and statistical manual of mental disorders, Fourth Edition, Text Revision (DSM-IV-TR ${ }^{\circledR}$ )

- Autism Society of America (2006) Information retrieved from website: http://www.autism society.org/site/PageServer?pagename=FactsStats

- Ayres, A. J., \& Tickle, L.S. (1980). Hyperresponsivity to touch and vestibular stimuli as a predictor of positive response to sensory integration procedures by autistic children. American Journal of Occupational Therapy, 34, 375-381

- Baranek, G.T. (1999a). Autism during infancy: A retrospective video analysis of sensorymotor and social at 9-12 months of age. Journal of Autism and Developmental Disorders, 29, 213-224.

- Baranek, G.T., David, F.J., Poe, M.D., Stone, W.L., and Watson, L.R. (2006). Sensory Experiences Questionnaire: discriminating sensory features in young children with autism, developmental delays, and typical development. Journal of Child Psychology and Psychiatry 47:6, pp 591-601.

- Baranek, G.T., Foster, L.G., \& Berkson, G. (1997). Sensory defensiveness in persons with developmental disabilities. Occupational Therapy Journal of Research, 17, 173185.

- Barry, R. J., and James, A. L. (1988). Coding of stimulus parameters in autistic, retarded and normal children: Evidence for a two-factor theory of autism. International Journal of Psychophysiology, 6, 139-149.

- Behrmann, M., Thomas, C., and Humphreys, K. (2006) Seeing it differently: visual processing in autism. Trends in Cognitive Sciences. 10(6): 258-64.

- Bemporad, J. R. (1979). Adult recollections of a formerly autistic child. Journal of Autism and Developmental Disorders, 9(2) 179-197

- Cambridge Scientific Abstracts (2007). Proquest-CSA, LLC.

- Cesaroni, L., \& Garber, M. (1991). Exploring the experience of autism through first hand accounts. Journal of Autism and Developmental Disorders, 21(3), 303-313. 
- Corbett, B.A., Mendoza, S., Abdullah, M., Wegelin, J.A., Levine, S. (2006). Cortisol circadian rhythms and response to stress in children with autism. Psychoneuroendocrinology, 31(1): 59-68.

- Davis, R.A., Bockbrader, M.A., Murphy, R.R., Hetrick, W.P., and O'Donnell B.F., (2006). Subjective perceptual distortions and visual dysfunction in children with autism. Journal of Autism \& Developmental Disorders. 36(2): 199-210.

- Dawson, G. \& Watling, R. (2000) Interventions to Facilitate Auditory, Visual, and Motor Integration in Autism: A Review of the Evidence. Journal of Autism and Developmental Disorders. 30 (5), 1573 -

- Dunn, W. (1994). Performance of typical children on the sensory profile: An item analysis. American Journal of Occupational Therapy, 48, 967-974.

- Dunn, W. (1999). Sensory profile. San Antonio, TX: The Psychological Corporation.

- Eldenson, S.M., Edelson, M.G., Kerr, D.C.R. and Granding, T. (1999). Behavioral and physiological effects of deep pressure on children with autism: A pilot study evaluating the efficacy of Grandin's Hug Machine. American Journal of Occupational Therapy, 53, 145-152.

- $\quad$ Eric (2007). Education Resources Information Center Database. United States of America (USA).

- Fertel-Daly, D., Bedell, G., Hinojosa, J., (2001). Effects of a weighted vest on attention to task and self-stimulatory behaviors in preschoolers with pervasive developmental. American Journal of Occupational Therapy,

- Gerland, G. (2003). A real person: Life on the outside. London: Souvenir Press.

- Grandin, T., and Scariano, M. (1986). Emergence: Labeled autistic. Novato, CA: Arena

- Gomot, M., Bernard, F.A., Davis, M.H., Belmonte, M.K., Ashwin, C., Bullmore, E.T., and Baron-Cohen, S. (2006). Change detection in children with autism: an auditory event related fMRI study. Neuroimage, 29(2): 475-84.

- Gravel, J.S., Dunn, M., Lee, W.W., and Ellis, M.A. (2006). Peripheral audition of o children on the autistic spectrum. Ear \& Hearing. 27(3): 299-312.

- Greenspan, S. \& Wieder, S. (2006). Engaging autism: Using the Floortime approach to help children relate, communicate, and think. Cambridge, MA: Perseus Books.

- Greenspan, S. \& Wieder, S. (1997). Developmental patterns and outcomes in infants and children with disorders in relating and communicating. A chart review of 200 cases of children with autistic spectrum diagnoses. Journal of Developmental and Learning Disorders, 1, 87-141.

- Happe, F. R. G. (1995). “'Autism: 'Theory of mind' and beyond”. In Psychological perspectives in autism. Autism Research Unit, University of Sunderland, United Kindgdom.

- Harrison, J. and Hare, D.J. (2004) Brief Report: Assessment of Sensory Abnormalities in People with Autistic Spectrum Disorders. Journal of Autism and Developmental Disorders, Vol. 34, No. 6.

- Herbert, M. (2005). Autism, a brain disorder or a disorder that affects the brain? Clinical Neuropsychiatry, 2, 354-369.

- Hirstein, W., Iversen, P., \& Ramachandran, V. S. (2001). Autonomic responses of autistic children to people and objects. Proceedings of the Royal Society of London. B, Biological Sciences, 268, 1883-1888.

- James, A. L., \& Barry, R. J. (1984). Cardiovascular and electrodermal responses to simple stimulation autistic, retarded and normal children. International Journal of Psychophysiology, 1, 179-193. 
- Johnson-Ecker, C. L., and Parham, L. D. (2000). The Evaluation of Sensory Processing: A validity study using contrasting groups. American Journal of Occupational Therapy, 54, 494-503.

- Jones, R. S. P., Quigney, C., \& Huws, J. C. (2003). First-hand accounts of sensory perceptual experiences in autism: A qualitative analysis [Electronic version].

- Journal of Intellectual \& Developmental Disability, 28(2), 112-121.

- Kanner, L. (1943). Autistic disturbances of affective contact. Nervous Child, 2, 217- 250.

- Karsteadt, A.E. (1983). The effects of vestibular stimulation on verbalization and attending behavior of an autistic child. Sensory Integration Special Interest Newsletter, 6(1), 4.

- Kasai, K., Hashimoto, O., Kawakubo, Y., Yumoto, M., Kamio, S., Itoh, K., Koshida, I., Iwanami, A., Nakagome, K., Fukuda, M., Yamasue, H., Yamada, H., Abe, O., Aoki, S., Kato, N. (2005). Delayed automatic detection of change in speech sounds in adults with autism: a magnetoencephalographic study. Clinical Neurophysiology, 116(7): 1655-64.

- Kern, J.K.,Trivedi, .H., Garver, C.R., Grannemann, B.D., Andrews, A.A., Savla, J.S., Johnson, G.S., Jyutika A. Mehta, J.A., Schroeder, J.L. (2006). The pattern of sensory processing abnormalities in autism. Autism, 10, 480.M

- Kientz, M. A., and Dunn, W. (1997). A comparison of the performance of children with and without autism on the Sensory Profile. American Journal of Occupational Therapy, 51, 530-537.

- Kinsbourne, M. (1987). Cerebral-brainstem relations in infantile autism. In E. Schopler \& G. Mesibov (Eds.), Neurobiological issues in autism (pp. 107-125). NewYork: Plenum Press.

- Leekam, S.R., Nieto, C., Libby, S.J., Wing, L., and Gould, J. (2006). Describing the Sensory Abnormalities of Children and Adults with Autism. Journal of Autism and Developmental Disorders.

- Lepisto, T., Kujala, T., Vanhala, R., Alku, P., Huotilainen, M., Naatanen, R. (2005). The discrimination of and orienting to speech and non-speech sounds in children with autism. Brain Research. 1066(1-2):147-57.

- Linderman, T.M. and Steward, K.B. (1999). Semsory integrative based occupational therapy and functional outcomes in young children with pervasive developmental disorders: A single subject study. American Journal of Occupational Therapy, 53, 207213.

- Liss, M., Saulnier, C., Fein, D., and Kinsbourne, M. (2006). Sensory and attention abnormalities in autistic spectrum disorders. Autism, 10(2), 155-172.

- Mandell, D. Novak, M. \& Zubrisky, C. (2006). Factors associated with age of diagnosis

- $\quad$ among children with autism spectrum disorders. Pediatrics. 116 (6): 1480 -1486.

- McAlonan, G.M., Daly, E., Kumari, V., Critchley, H.D., van Amelsvoort, T., Suckling, J. et al. (2002). Brain anatomy and sensorimotor gating in Asperger's syndrome. Brain, 125(Pt 7), 1594-1606.

- Miller, L. J., McIntosh, D. N., McGrath, J., Shyu, V., Lampe, M., Taylor, A. K., et al. (1999). Electrodermal responses to sensory stimuli in individuals with fragile $X$ syndrome: a preliminary report. American journal of medical genetics, 83(4):268-79.

- Miller, L. J., Reisman, J. E., McIntosh, D. N., and Simon, J. (2001). An ecological model of sensory modulation: Performance of children with fragile $\mathrm{X}$ syndrome, autistic disorder, attention-deficit/hyperactivity disorder, and sensory modulation dysfunction. In S.S. Roley, E.I. Blanche, and R.C. Schaaf (Eds.), Understanding the nature of sensory integration in diverse populations (pp. 57-82). San Antonio, TX: Psychological Corporation. 
- Minshew, N.J., Sung, K., Jones, B.L., and Furman, J.M. (2004). Underdevelopment of the postural control system in autism. Neurology. 63(11):2056-61.

- Miyazaki M, Fujii E, Saijo T, Mori K, Hashimoto T, Kagami S, Kuroda Y. Short-latency somatosensory evoked potentials in infantile autism: evidence of hyperactivity in the right primary somatosensory area. Dev Med Child Neurol. 49(1):13-7.

- Mostofsky, S.H., Dubey, P., Jerath, V.K., Jansiewicz, E.M., Goldberg, M.C., Denckla, M.B. (2006). Developmental dyspraxia is not limited to imitation in children with autism spectrum disorders. Journal of the International Neuropsychological Society. 12(3):31426.

- $\quad$ OT Seeker (2007). The Occupational Therapy Systematic Evaluation of Evidence.

- OT Search (2007). The Occupational Therapy Bibliographic System. The Wilma West Occupational Therapy Library. The American Occupational Therapy Association and The American Occupational Therapy Foundation.

- O’Riordan, M and Passetti, F. (2006) Discrimination in Autism Within Different Sensory Modalities. Journal of Autism and Developmental Disorders, 36:665-675

- Ornitz, E.M. (1989). Autism at the interface between sensory and information processing. In G. Dawson (Ed.), Autism: Nature, diagnosis, and treatment (pp. 174-207). New York: Guilford Press.

- Parham, L.D., and Ecker, C. (2002). Evaluation of Sensory Processing. University of Southern Califonia, Los Angeles, California. United States.

- $\quad$ Ray, T., King, L.J. and Grandin, T. (1988). The effectiveness of self-initiated vestibular stimulation in producing speech sounds in an autistic child. Occupational Therapy Journal of Research,8(3) 186-190.

- Rogers, S. J. and Ozonoff, S. (2005). Annotation: What do we know about sensory dysfunction in autism? A critical review of the empirical evidence. Journal of Child Psychology and Psychiatry 46:12 (2005), pp 1255-1268.

- Rogers, S.J., Hepburn, S., \& Wehner, E. (2003). Parent reports of sensory symptoms in toddlers with autism and those with other developmental disorders. Journal of Autism and Developmental Disorders, 33, 631-642.

- Rosenhall, U., Nordin, V., Sandstroem, M., Ahlsen, G., \& Gillberg, C. (1999). Autism and hearing loss. Journal of Autism and Developmental Disorders, 29, 349-357.

- Rubenstein, J.L., \& Merzenich, M.M. (2003). Model of autism: Increased ratio of excitation/inhibition in key neural systems. Genes Brain and Behavior, 2, 255-267.

- Samson, F., Mottron, L., Jemel, B., Belin, P., and Ciocca, V., (2006). Can spectrotemporal complexity explain the autistic pattern of performance on

- $\quad$ auditory tasks?. Journal of Autism \& Developmental Disorders. 36(1): 65-76.

- Scharre, J., \& Creedon, M. (1992). Assessment of visual function in autistic children. Optometry and VisionScience, 69, 433-439.

- Schieve, L.A., Blumberg, S.J., Rice, C., Visser, S.N, and Boyle, C. (2007). The relationship between autism and parenting stress. Pediatrics, 119 Suppl 1 :S114-21

- Smith, S.A, Press, B., Koenig, K.P., Kinnealey, M. (2005). Effects of sensory integration intervention on self-stimulating and self-injurious behaviors. American Journal of Occupational Therapy. 59(4): 418-25.

- $\quad$ Stone, W.L., \& Hogan, K.L. (1993). A structured parent interview for identifying young children with autism. Journal of Autism and Developmental Disorders, 23, 639-652.

- Talay-Ongan, A., \& Wood, K. (2000). Unusual sensory sensitivities in autism: A possible crossroads. Inter-national Journal of Disability Development and Education, 47, 201212. 
- Tharpe, A.M., Bess, F.H., Sladen, D.P., Schissel, H., Couch, S., and Schery, T. (2006) Auditory characteristics of children with autism. Ear \& Hearing. 27(4):430-41.

- Tecchio, F., Benassi, F., Zappasodi, F., Gialloreti, L.E., Palermo, M., Seri, S. et al. (2003). Auditory sensoryprocessing in autism: A magnetoencephalographic study. Biological Psychiatry, 54, 647-54.

- Tomchek, S. \& Dunn, W. (2007). Sensory Processing in Children With and Without Autism: A Comparative Study Using the Short Sensory Profile. American Journal of Occupational Therapy, 61(2).

- The Action Potential Corporation (2007). Manual, Alternative, and Natural Therapy Index Systems (Mantis).

- United States National Library of Medicine (2007). Document retrieved from website http://www.nlm.nih.gov/

- Volkmar, F., Chawarska, K., \& Klin, A. (2005). Autism in infancy and early childhood. Annu. Rev. Psycholo. 56: 315 -336.

- Waterhouse, L., Fein, D., \& Modahl, C. (1996). Neuro-functional mechanisms in autism. Psychological Review, 103, 457-489.

- Williams, D. (1994). Somebody somewhere. New York: Doubleday.

- Williams, D. (1999). Autism and sensing: The unlost instinct. London: Jessica Kingsley Publishers.Williams, 1994

- Zisserman, L. (1992). The effects of deep pressure on self-stimulating behaviors in a child with autism and other disabilities. American Journal of Occupational Therapy, 46, 547-551. 